\title{
Solid Phase Micro-Extraction, a Versatile and Handy Tool in Environmental Trace Organic Analysis, Gets a New Class of Coatings, Polymeric Ionic Liquids
}

\author{
Marta Wasielewska, Anna Banel, and Bogdan Zygmunt
}

\begin{abstract}
Solid phase microextraction (SPME) fundamentals, characteristics and application are described. The special stress is put on characteristics of sorbents used as SPME fiber coatings. Description of commercially available fibers and the present-day trends in development of new sorbents for SPME are given. Discussion is focused on possibility of application of ionic liquids (IL) and polymeric ionic liquids (PIL) as SPME fiber coatings. An example of application of PIL coating to determine volatile fatty acids in water is given.
\end{abstract}

Index Terms-Environmental trace organic analysis, chromatography, solid phase microextraction, ionic liquids, polymeric ionic liquids.

\section{INTRODUCTION}

Environmental pollution studies and quality assessment require determination of pollutants in the media discharged into the environment as well as in environmental compartments. Many organic pollutants are so harmful that they must be monitored at very low concentration in very complex samples. Such analytical tasks require reliable analytical procedures characterized by low limits of determination. The techniques of high sensitivity, selectivity and identification power, generally preceded by sample preparation must be used.

To determine trace organic pollutants usually the separation techniques such as gas, liquid and supercritical chromatography and capillary electrophoresis are employed. Sample preparation, most often a necessary step, is generally based on selective isolation and enrichment of analytes from an original sample. This step is generally labour and time consuming, considerably contributes to results uncertainty and can have the most harmful effect on the environment. So organic solvent-free sample preparation techniques have been looked for. Examples of the techniques are: solid phase microextraction - SPME, stir bar sorptive extraction - SBSE, single drop microextraction - SDME, microextraction in a packed syringe - MEPS, etc.

SPME is simple and versatile; the instrument used for the analysis proper does not need any modification. The technique can be relatively sensitive and selective and is widely used in trace organic analysis and the range of its application is rapidly increasing [1].

Manuscript received December 8, 2012; revised February 12, 2013.

Bogdan Zygmunt is with the Gdansk University of Technology (e-mail: bogdan.zygmunt@pg.gda.pl).

\section{A. Basics of Solid Phase Micro-Extraction (SPME)}

An SPME manual is a simple device whose design is based on a micro-syringe technology. A thin fused silica fiber coated with the sorption medium is attached to the syringe plunger. The fiber can be withdrawn from the needle for exposition to the sample and desorption of analytes and pulled back into the needle for transport and whenever the needle is to be pierced through a septum. In the injection port of chromatograph the fiber is withdrawn out, the analytes are thermally desorbed for GC analysis or with solvent for LC. Mobile phase carries analytes to the chromatographic column.

Analytes are extracted to the fiber coating: a) directly from gas or liquid by immersing the fiber in the medium studied; $b$ ) from the headspace (gas phase above the condensed phase, the phases at equilibrium) of liquid or solid to be analysed; and c) from the space separated from the medium studied by semipermeable membrane. Enrichment factors and selectivity coefficients, depend, first of all, on coating material.

The typical fiber coatings can be classified into two groups [1]: a) homogeneous polymers such as nonpolar polydimethylsiloxane (PDMS), polar polyacrylate (PA), and polar Carbowax and b) composite extraction media consisting of a partially cross-linked polymer embedded with porous particles of a second component (PDMS/divinylbenzene (DVB), Carbowax/ DVB, PDMS/Carboxen, Carbowax/templated resin, etc). Basing on the commercial fibers a lot of analytical procedures have been developed and thousands of analyses conducted. The technique is so convenient and handy that research is made to extend the range of application with respect to matrices, analytes, especially polar, and quality of the results. This is to be achieved by producing the fibers of increased thermal and chemical stability as well as of higher extraction efficiency, selectivity, and even of task specific properties. The new polymers are: 1) Nafion (highly polar); 2) Polypyrrole; 3) molecularly imprinted polymers; and 4) sol-gel hybrid organic-inorganic polymers (sol-gel PDMS, PEG, crown ether, dendrimer). In recent years, ionic liquids (ILs) have drawn considerable attention of analytical chemistry community, including SPME specialists.

\section{B. Ionic Liquid Extractants}

Ionic liquids (ILs) first described by Walden in 1914 [2] are characterized by low melting points, (generally, not higher than $100{ }^{\circ} \mathrm{C}$ ) and very low vapour pressure; they are stable in the air in a wide temperature range and their viscosity and miscibility with water can be tuned. Up to now 
more than 200 ILs have been described and their number rapidly increases. A typical ionic liquid is an organic salt composed of organic cation (alkylimidazolium, alkylammonium, alkylphosphonium, alkylpirydynium, alkylpirolydynium) and organic or inorganic anion, most often hexafluorophosphate, bis[(trifluoromethyl)sulphonyl]imide, perfluoroalkylsulphate, chloride, etc. Examples of cations and anions which form an ionic liquid are shown in Table I.

TABLE I: EXAMPLES OF CATIONS AND ANIONS FORMING IONIC LIQUID STRUCTURES

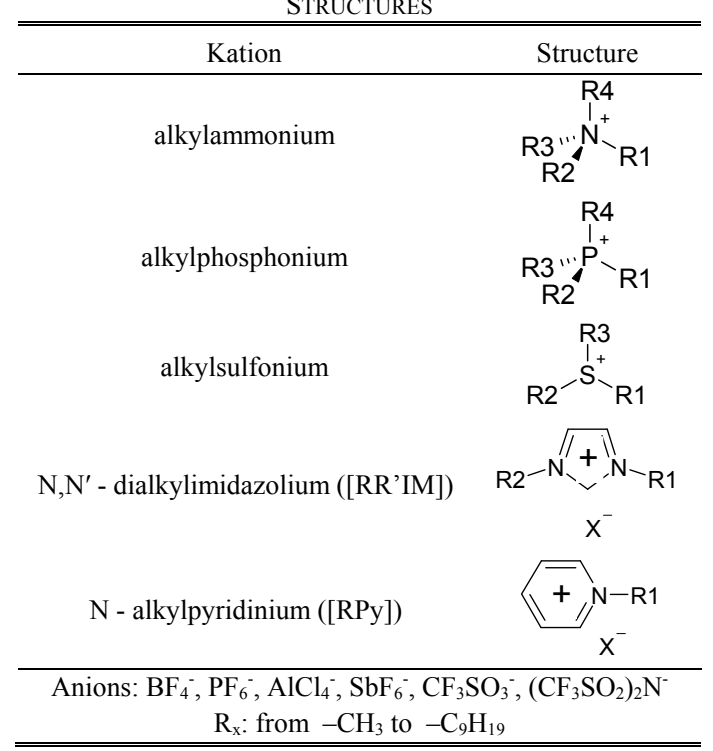

Selecting anion and cation structure and anion-cation combinations, properties of ILs such as viscosity, density, water miscibility, selectivity and extraction capability can be programmed [3]. The effect of anion on the IL properties is shown in Table II.

Due to high density, two-phase systems formed with water or with organic solvent can easily and fast be separated. Negligible vapour pressure minimizes environmental pollution resulting from solvent evaporation and permits the application of vacuum systems. Asymmetry and delocalization of charge make vibrations easier which results in longer distances between charges, decreased stability of crystal lattice and lowered melting points. Due to high boiling points, low vapour pressure in a wide temperature range and thermal stability, even at temperature above $250^{\circ} \mathrm{C}$, some ILs can used as stationary phases in gas chromatography. High solvation strength makes that ILs are good solvents for the wide spectrum of organic compounds and have been applied as extractants in liquid-liquid extraction (LLE), dispersive LLME, single drop micro-extraction (SDME) and hollow fiber liquid phase microextraction (HF-LPME).

\section{Ionic Liquids as SPME Fiber Coatings}

Due to the physical and chemical properties and possibility of their tuning ILs could be applied as the SPME fiber coatings.

First they were used as coatings for headspace (HS)-SPME extraction in determination of BTEX in paints. The coating was 1-octyl-3-methylimidazolium hexafluorophosphate, coated on a bare silica fiber. It was removed after each run hence carryover was avoided. To increase the stability the fiber was pre-coated with Nafion. The other ways of increasing stability were [4]: chemical bonding of ILs to fused silica fibers, preparation of IL-mediated sorbent coatings, and sol-gel derived IL-bonded sorbent coatings.

TABLE II: COMPARISON OF SELECTED PROPERTIES OF POLYMERIC IONIC LIQUIDS

\begin{tabular}{|c|c|c|c|c|c|c|}
\hline \multirow{2}{*}{ Kation } & \multirow{2}{*}{ Anion } & \multicolumn{3}{|c|}{ Solubility } & \multirow{2}{*}{ Viscosity } & \multirow{2}{*}{ Thermal Stability } \\
\hline & & $\mathrm{H}_{2} \mathrm{O}$ & Aceton & Ethyl acetate & & \\
\hline \multirow{4}{*}{ poli(1-vinyl-3-etyloimidazolium) } & $\mathrm{Br}^{-}$ & + & - & - & Low & Very high \\
\hline & $\mathrm{BF} 4^{-}$ & - & + & - & High & High \\
\hline & {$\left[\mathrm{CF}_{3} \mathrm{SO}_{2}\right]_{2} \mathrm{~N}$} & - & + & - & Very high & Low \\
\hline & {$\left[\mathrm{CF}_{3} \mathrm{CF}_{2} \mathrm{SO}_{2}\right]_{2} \mathrm{~N}$} & - & + & + & Very high & Low \\
\hline
\end{tabular}

\section{Polymeric Ionic Liquids as SPME Fiber Coatings}

The great step forward in increasing stability of IL based coatings while keeping their main advantages was introducing polymeric ionic liquids (PILs) by Anderson and co-workers [5]. These new SPME coatings show higher viscosity and thermal stability which prevents coating flow into a gas chromatograph during thermal desorption of analytes. Interaction of hydrophobic fragments of a given PIL with the fiber is often sufficiently strong that it is possible to extract analytes to the fiber directly from the aqueous medium. Lopez-Darias et al [6] were the first who extracted analytes from aqueous media by direct immersing the fiber in the sample. In their work polyaromatic hydrocarbons and substituted phenols were extracted from water into poly ([ViHDIm][ $\left.\left.\mathrm{NTf}_{2}\right]\right)$ coating [6].

Thermal stability of the coating depends on the structure and composition of anion and cation. PILs with imidiazolium cations have high thermal stability increasing with alkyl group length. However, the anion has much higher effect on stability which is generally higher for organic than halogen anions.

The reverse can be observed for extraction efficiency. Meng et al [7] compared extraction of volatile fatty acids using poly (1-vinyl-3-hexylimidazolium) chloride, poly ([ViHIm] [Cl]) and poly (1-vinyl-3-hexylimidazolium) bis[(trifluoromethyl)sulphonyl]imide, poly([ViHIm][ $\left.\left.\mathrm{NTf}_{2}\right]\right)$. The former exhibits high hydrogen-bond basicity and polarity and hence higher extraction efficiency while the latter higher thermal stability [7].

To show possibility of some property tuning Meng and Anderson synthesized two PILs, i.e. poly(1-4-vinylbenzyl)-3-hexadecylimidazolium (bis[(tri- 
fluoromethyl)sulphonyl]imide and poly (1-vinyl3-hexylimidazolium) bis[(trifluoromethyl)sulphonyl] imide and studied their extraction efficiency with respect to PAHs. Due to interactions the former exhibits higher extraction efficiency as expected.

\section{EXEMPLARY APPLICATION OF PIL [POLY}

\section{(1-VINYL-3-HEXYLIMIDAZOLIUM) CHLORIDE] COATING} SPME- GC-MS TO DETERMINE SHORT CHAIN MONOCARBOXYLIC ACIDS

As shown by Meng et al. [8] PIL coated SPME fibers can be used to isolate and introduce polar organic compounds into a GC column. In this work an attempt was made to use GC-MS preceded by poly(ViHImCl) SPME extraction to determine some VFAs in the aqueous sample.

\section{A. Instrumentation}

The homemade SPME device described elsewhere [5] was received from prof. J. Anderson research group from University of Toledo (USA). It was made out of the $5 \mu \mathrm{l}$ syringe (Hamilton, Reno, NV, USA) in which the stainless thin fiber attached to a plunger was replaced by a fused silica capillary glued to the top of the plunger. Polyimide film was burned off out of $1 \mathrm{~cm}$ capillary whose end was sealed using a microflame torch. Such prepared fiber was washed with four solvents: acetone, hexane, methanol and methylene chloride. The bare fiber segment was coated with poly(ViHImCl) according to procedure briefly described elsewhere [5]. The fiber was conditioned for $5 \mathrm{~min}$ in the GC injector at the desorption temperature.

Chromatographic analysis was carried out using a Thermo Finnigan Trace GC gas chromatograph equipped with a split/splitless injector coupled with a TRACE DSQ quadrupole mass spectrometer. VFAs were desorbed from the fiber in a gas chromatograph splitless injection port with a $0.75 \mathrm{~mm}$ inner diameter liner. The injector temperature was $170{ }^{\circ} \mathrm{C}$ for poly $\left(\mathrm{ViHIm}^{+} \mathrm{Cl}^{-}\right)$fiber. A Restek Stabilwax (cross-bond PEG treated with nitroterephthalic acid) open tubular capillary column $(30 \mathrm{~m} \times 0.25 \mathrm{~mm} \times 0.50 \mu \mathrm{m})$ was used for separation. The helium at $1.0 \mathrm{~mL} / \mathrm{min}$ was used as a carrier gas. The oven temperature was programmed: from $40{ }^{\circ} \mathrm{C}$ for $3 \mathrm{~min}$, then $60{ }^{\circ} \mathrm{C} / \mathrm{min}$ to $130{ }^{\circ} \mathrm{C}(1 \mathrm{~min})$, and then $10{ }^{\circ} \mathrm{C} / \mathrm{min}$ until $230{ }^{\circ} \mathrm{C}$ holding the final temperature for 1 min. The temperature of the transfer line and that of the electron impact ion source $(70 \mathrm{eV})$ was $240^{\circ} \mathrm{C}$. MS was operated in a full spectrum scanning mode $(\mathrm{m} / \mathrm{z} 40-400)$ and in selected ion monitoring (SIM) mode. The ions selected for monitoring acids were as follows: ethanoic-43, 45, 60; propanoic-45, 57, 74; methylpropanoic-43, 73, 88; n-butanoic-60, 73; 3- methylbutanoic-41, 60, 87; n-pentanoic-60, 73, 87; n-hexanoic- 60, 73, 87; n-heptanoic-41, 60, 73 .

\section{B. HS-SPME Analysis}

Aqueous samples of $8 \mathrm{~mL}$ in volume were poured out into the $15 \mathrm{~mL}$ headspace amber vial, a magnetic stir bar was introduced and $\mathrm{NaCl}$ was added. The vial was closed with a screw cap and $\mathrm{pH}$ was adjusted to about 2 by injecting $\mathrm{H} 2 \mathrm{SO} 4$. The SPME fiber was exposed for $10 \mathrm{~min}$ to the headspace of the sample being stirred at $1200 \mathrm{rpm}$. Extraction temperature was kept at $35^{\circ} \mathrm{C}$. After the prefixed time the fiber was removed from the sample vial and immediately inserted into the heated GC injector for thermal desorption (4 $\min , 170^{\circ} \mathrm{C}$ ) and analysis. The chromatogram obtained for a standard sample containing all acids at a concentration of 25 $\mathrm{mg} / \mathrm{L}$ each is given in Fig. 1.

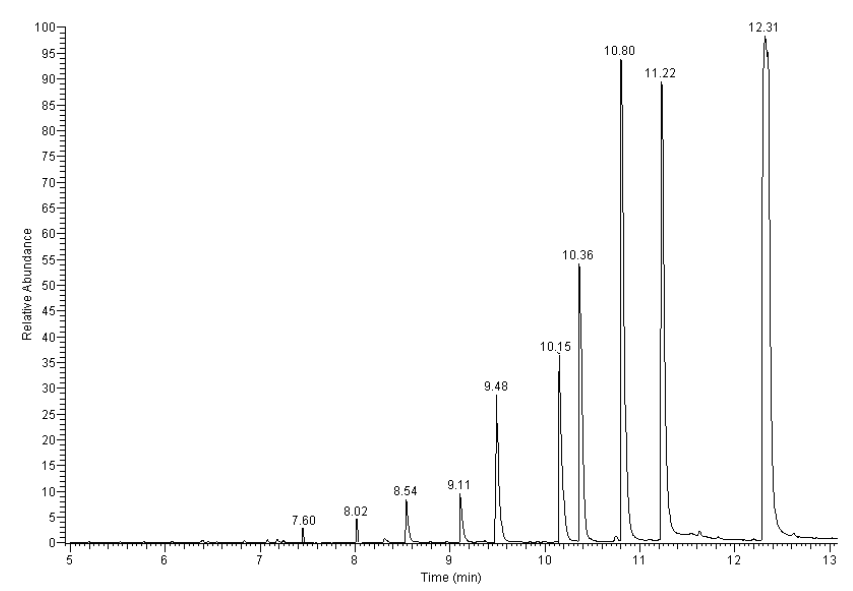

Fig. 1. The GC-MS chromatogram of analytes extracted to SPME fiber from headspace of a VFA standard aqueous solution and thermally desorbed in an injection port. Retention times: 7.60 - acetic acid, 8.02 - propionic acid, 8.54 - isobutyric acid, 9.11 - butyric acid, 9.48 - isovaleric acid, 10.15 valeric acid, 10.80 - isohexanoic acid, 11.22 - hexanoic acid, 12.31 heptanoic acid

\section{CONCLUSION}

SPME is so convenient and handy sampling and sample preparation technique that studies are conducted to widen their application and improve quality of data produced mainly by introducing new sorbent coatings. Recently, ionic liquids have been examined as possible SPME fiber coatings. Since their properties can be tuned very selective and even task specific coatings can be produced. Thermal stability of coating is a limiting factor for using SPME in combination with GC and different approaches have been used to form more thermally and chemically stable coatings. First physically bonded IL coatings applicable to extract analytes from HS were formed. Then chemically bonded, IL mediated, and sol-gel derived IL SPME sorbent coating were produced. A great step forward was achieved by introducing polymeric ionic liquids (PILs) by researchers of Department of Chemistry, University of Toledo, Toledo, OH, USA. PIL-based coatings are characterized by increased thermal and chemical stability and can be used for extraction from HS as well as in direct immersion SPME. The large number of PIL coatings can be synthesized with properties tuned to selectively isolate and introduce to a chromatographic column a given class of analytes or even a given analyte. However, up to now the limited number of IL/PIL based coatings have been examined and, in fact, those applied have some limitations concerning thermal desorption in the GC injector. The further studies should be conducted to design PILs which exhibit high thermal stability, superior selectivity for target analytes and which, at the same time, are resistant to extreme matrix conditions including high salt and organic modifier contents and low/high pH. Poly 
(1-vinyl-3-hexylimidazolium) chloride based coating can be used to isolate VFAs from HS of aqueous samples and to desorb them in GC injector for quantitative determination.

\section{ACKNOWLEDGMENT}

This work was supported by project: „The development of interdisciplinary doctoral studies at the Gdansk University of Technology in modern technologies" (Project No: POKL.04.01.01-00-368/09); Duration: $\quad 1.10 .2009$ $-30.09 .2015$

\section{REFERENCES}

[1] J. Pawliszyn and H. L. Lord, Handbook on Sample Preparation, John Willey \& Sons, New Jersey, Chapter 23, pp. 445-473, 2010.

[2] P. Walden, "Molecular weights and electrical conductivity of several fused salts," Bull. Acad. Sci. (St. Petersburg), pp. 405-422, 1914.

[3] Y. Meng and J. L. Anderson, "Tuning the selectivity of polymeric ionic liquid sorbent coatings for the extraction of polycyclic aromatic hydrocarbons using solid-phase microextraction," J. Chromatogr. A, vol. 1217, pp. 6143-6152, 2010.

[4] T. D. Ho, A. J. Canestraro, and J. L. Anderson, "Ionic liquids in solid-phase microextraction: A review," Analytica Chimica Acta, vol. 695, pp. 18-43, 2011.

[5] F. Zhao, Y. Meng, and J. L. Anderson, "Polymeric ionic liquids as selective coatings for the extraction of esters using solid-phase microextraction," J. Chromatogr. A, vol. 1208, pp. 1-9, 2008.

[6] J. L. Darias, V. Pino, J. L. Anderson, C. M. Graham, and A. M. Afonso, "Determination of water pollutants by direct-immersion solid-phase microextraction using polymeric ionic liquid coatings," J. Chromatogr. $A$, vol. 1217, pp. 1236-1243, 2010.

[7] Y. Meng, V. Pino, and J. L. Anderson, "Role of counteranions in polymeric ionic liquids-based solid-phase microextraction coatings for the selective extraction of polar compounds," Anal. Chim. Acta, vol. 687, 141-149, 2011.

[8] Y. Meng, V. Pino, and J. L. Anderson, Analytical Chemistry, vol. 81, pp. 7107-7112, 2009 .

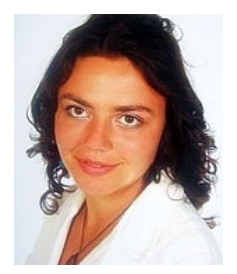

Marta Wasielewska was born in Gniew, Poland, in 1984. She received M.Sc. Eng. degree in Technologies of Environmental Protection (specialization: Monitoring and Analytics of Environmental Pollution) at Chemical Faculty, Gdansk University of Technology in Poland in 2009. The final thesis was devoted to development headspace - solid phase microextraction - gas chromatography - mass spectrometry detection method for determination of the volatile fatty acids. In the same year Marta Wasielewska started $\mathrm{PhD}$ degree studies at the Department of Analytical Chemistry in Gdańsk University of Technology in Poland. She spent three months $(06-09.2011)$ at the University of Toledo, Ohio, US during realization of project: Development of solid-phase microextraction sorbent coatings based on bonded polymeric ionic liquids for the selective extraction and determination of volatile fatty acids.

She is an author/ co-author of 5 articles in reviewed scientific journals, 10 articles in conference's materials, 12 presentations and 3 posters during polish and international conferences.

M. Sc. Eng. Wasielewska is conducting the $\mathrm{PhD}$ research within the framework of the research project no 2012/05/N/ST4/02026 (founded by the National Science Center, Poland).

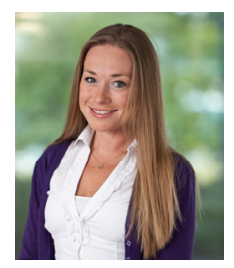

Anna Banel was born in Gdansk, Poland in 1980. She received M.Sc. Eng. degree in Technologies of Environmental Protection (specialization: Monitoring and Analytics of Environmental Pollution) at Chemical Faculty, Gdansk University of Technology in Poland in 2005. The final thesis was devoted to investigation of the heavy metals levels in nails and hair of workers of a slide bearings factory. In 2006 Anna Banel started PhD degree studies at the Department of Analytical Chemistry in Gdańsk University of Technology in Poland. Anna Banel has graduated in 2011; she has defended her $\mathrm{PhD}$ thesis devoted to development and comparison of the gas chromatography methods for determination of the volatile fatty acids. At the beginning of October 2011, Anna Banel started a post doc in the Norwegian Institute for Air Research (NILU, Kjeller, Norway).

During the last semester of study she went for student exchange to Delft (Netherlands) within the framework of SOCRATES/ERASMUS program. In 2005 she worked in the Voivodship Station of Sanitary and Epidemiology at Laboratory for environmental studies Department in Gdańsk, Poland. She is an author/ co-author of 1 book chapter, 10 articles in reviewed scientific journals, 8 articles in conference's materials, 16 presentations and 8 posters during polish and international conferences.

Dr Anna Banel was a laureate of "Inno-Doktorant" Scholarship for PhD students, II edition, founded by the European Union within the framework of the European Social Fund in 2009. The PhD research has been conducted within the framework of the research project no N N523 230535 (founded by the Polish Ministry of Science and Higher Education).

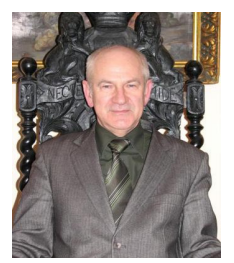

Bogdan Zygmunt was born in Poland (Lublin province) on 3rd March 1946. He graduated from Chemical Faculty, Gdansk University of Technology (GUT), Poland in 1969 receiving MSc degree, engineer in the area of Chemical Technology of Inorganic Compounds. The successive degrees were also granted by GUT: in $1976 \mathrm{PhD}$ (determination of organic sulfur compounds in petroleum products), in 1997 DSc (development of methods to determine trace organic environmental pollutants - main field of research) and title of professor in 2006. Bogdan Zygmunt has been working (research and teaching) at Gdansk University of Technology since 1969 up to now. He spent one year as visiting scientist at the Free University of Amsterdam (1981-82); 3 years as an assistant professor at Garyounis University in Benghazi (Libya) (1985-88). Short time visits included: University of Hamburg (Germany), University of Waterloo (Canada), Swedish Environmental Research Institute (Sweden).

His research activities included development of new procedures of: determination of trace thiols in petroleum products; isolation and enrichment and chromatographic determination of trace organic pollutants in air, water and soil. He was an author or co-author of over 80 papers and over 100 communications, posters and conference lectures and also 12 chapters in scientific books; co-ordinator of projects granted by Polish Committee of Research and partner in three EC projects within Framework V and in one NATO project. He participated in staff mobility within Erasmus projects and also as a program manager in 2 Erasmus Mundus projects.

Prof. Zygmunt was a member of Organizing and Scientific Committees of international conferences in Poland; member of Commission of Environmental Analysis and Commission on Analytical Reagents and Reactions, Committee of Analytical Chemistry, Polish Academy of Sciences; member of Polish Chemical Society. He was awarded many times by Rectors of GUT and once by Ministry of Research and Higher Education, Poland. 